\title{
Turbulence Simulation on Zonal Flow Formations in the Presence of Parallel Flows ${ }^{*}$
}

\author{
Makoto SASAKI ${ }^{1,2,3)}$, Naohiro KASUYA ${ }^{1,2)}$, Yusuke KOSUGA ${ }^{1,2)}$, Tatsuya KOBAYASHI ${ }^{4}$, \\ Takuma YAMADA ${ }^{2,5)}$, Hiroyuki ARAKAWA ${ }^{6)}$, Shigeru INAGAKI ${ }^{1,2)}$ and Kimitaka ITOH ${ }^{2,4,7)}$ \\ ${ }^{1)}$ Research Institute for Applied Mechanics, Kyushu University, Kasuga 816-8580, Japan \\ ${ }^{2)}$ Research Center for Plasma Turbulence, Kyushu University, Kasuga 816-8580, Japan \\ ${ }^{3)}$ Department of Physics, Warwick University, Coventry CV4 7AL, United Kingdom \\ ${ }^{4)}$ National Institute for Fusion Science, Toki 509-5292, Japan \\ ${ }^{5)}$ Faculty of Arts and Science, Kyushu University, Fukuoka 819-0395, Japan \\ ${ }^{6)}$ Institute of Science and Engineering, Academic Assembly, Shimane University, Matsue 690-8504, Japan \\ ${ }^{7)}$ Institute of Science and Technology Research, Chubu University, Kasugai 487-8501, Japan
}

(Received 30 July 2019 / Accepted 12 September 2019)

\begin{abstract}
It is demonstrated that the zonal flow is controlled by the parallel flow in the cylindrical plasmas. A threedimensional turbulence simulation is performed, based on the reduced fluid model. The background parallel flow is applied by introducing the time independent parallel momentum source. Changing the magnitude of the momentum source, the behavior of the zonal flow is investigated. The drift wave turbulence is affected by the parallel flow, and it shows the spatial competition between turbulence modes. The spatial competition appears/disappears abruptly, depending on the intensity of the parallel momentum source. As a consequence of the transition between the turbulence state, the radial profile of the turbulence drastically changes, which leads to the change of the turbulence force to drive the zonal flow. In this way, the parallel flow indirectly affects the zonal flow through the deformation of the turbulence.
\end{abstract}

(C) 2019 The Japan Society of Plasma Science and Nuclear Fusion Research

Keywords: zonal flow, parallel flow, drift wave, turbulence competition, Reynolds force

DOI: $10.1585 /$ pfr.14.1401161

\section{Introduction}

Importance of flows in magnetized plasmas have been widely recognized. A helical flow is formed in magnetized plasmas, which consists of poloidal and toroidal flows. The poloidal flows, such as zonal flows [1] and the mean flow [2,3], contributes to the turbulence suppression due to their shears [4]. Recently, not only the shear of the flow but also the curvature of the flow has been reported to be important for the turbulence suppression [5-8]. On the other hand, the toroidal flow strongly affects the MHD stability [9], so that the driving mechanisms of the toroidal flow has been intensively investigated [10-12].

Poloidal and toroidal flows are not independent with each other. One of the flow coupling mechanism is the geometrical effect; the toroidicity connects each flow depending on the safety factor [13-15]. Another flow coupling process stems from the turbulence; both the poloidal and toroidal flows couple via turbulence, which is ubiquitous. For instance, the poloidal flow deforms the drift wave spectrum so that the toroidal momentum is generated [10]. The parallel flow shear driven instability, D' Angelo mode [16], which is often called the parallel velocity driven (PVG)

author'se-mail: sasaki@riam.kyushu-u.ac.jp

*) This article is based on the invited talk at the 35th JPSF Annual Meeting (2018, Osaka). mode [17-21] or Kelvin-Helmholtz instability [22], has been reported to drive the zonal flow [23], and the selection rule of the turbulence driven structures due to the parallel flow has been addressed [24]. Therefore, there is a possibility that the zonal flow could be controlled by the parallel flow, which leads to the controlling the plasma performance. In order to do that, the characteristics of the zonal flow driven the drift wave turbulence in the presence of the parallel flow should be investigated.

In this study, we demonstrate the zonal flow formation by the drift waves in the presence of parallel flows by using the turbulence simulation. The flow coupling process via turbulence is focused on by considering the cylindrical plasma (toroidal effect is ignored). Based on the reduced fluid model, the three-dimensional turbulence simulation is performed. The background parallel flow is introduced by applying the parallel momentum source. Changing the strength of the parallel momentum source, the behavior of the zonal flow is investigated. The zonal flow energy is found to be bifurcated due to the transition of the turbulence state. The rest of the paper is organized as follows. In Sec. 2, the brief summary of the turbulence simulation is described. The behavior of the zonal flow with controlling the parallel flow strength is discussed in Sec. 3. The summary is given in Sec. 4 . 


\section{Turbulence Simulation}

A three-dimensional simulation of turbulence in a cylindrical plasma in the presence of the helical flows is performed. We focus on a situation similar to basic plasma experiments, where the detail measurement of the turbulence is possible. The circular cross-section of plasma with a uniform axial magnetic field is considered. The model equation is based on the three-field reduced fluid equations [25],

$$
\begin{aligned}
& \frac{\partial N}{\partial t}+[\phi, N] \\
& =-D \nabla_{\|}^{2}(\phi-N)-D \nabla_{\|} N \nabla_{\|}(\phi-N) \\
& \quad-\nabla_{\|} V_{\|}-V_{\|} \nabla_{\|} N+\mu_{N} \nabla_{\perp}^{2} N+S_{n}, \\
& \frac{\partial \nabla_{\perp}^{2} \phi}{\partial t}+\left[\phi, \nabla_{\perp}^{2} \phi\right] \\
& =-D \nabla_{\|}^{2}(\phi-N)-D \nabla_{\|} N \nabla_{\|}(\phi-N) \\
& \quad-\nabla_{\perp} N \cdot \frac{d \nabla_{\perp} \phi}{d t}-v_{i n}\left(\nabla_{\perp}^{2} \phi+\nabla_{\perp} N \cdot \nabla_{\perp} \phi\right) \\
& \quad+\mu_{U} \nabla_{\perp}^{4} \phi, \\
& \frac{\partial V_{\|}}{\partial t}+\left[\phi, V_{\|}\right] \\
& =-V_{\|} \nabla_{\|} V_{\|}-\nabla_{\|} N-v_{i n} V_{\|}+\mu_{V} \nabla_{\perp}^{2} V_{\|}+S_{v},
\end{aligned}
$$

where $N=\ln \left(n_{e} / n_{0}\right), n_{e}$ is the electron density, $n_{0}$ is the density at plasma center $r=0$. Here, the electrostatic potential $\phi$ and the ion parallel velocity $V_{\|}$are normalized by the electron temperature $T_{e} / e$ and the sound speed, respectively, and the time and space are normalized by the ion cyclotron frequency and the ion sound Larmor radius, respectively. The particle and parallel momentum sources are introduced by $S_{n}$ and $S_{v}$. The parallel diffusivity of electrons is denoted by $D=A /\left(v_{e i}+v_{e n}\right), A$ is the ionelectron mass ratio, $v_{e i}$ is the electron-ion collision frequency, $v_{e n}$ is the electron-neutral collision frequency, $v_{i n}$ is the ion-neutral collision frequency, and $\mu_{N}, \mu_{U}, \mu_{V}$ are a set of viscosities. The boundary condition in the radial direction is set to $f_{m n}=0$ at $r=0, a$ for the modes with $m \neq 0$, and $\partial_{r} \boldsymbol{f}_{m n}=0$ at $r=0$ and $\boldsymbol{f}_{m n}=0$ at $r=a$ for the modes with $m=0$, where $f_{m n}=\left(\phi_{m n}, V_{\| m n}, N_{m n}\right)^{T}$.

A nonlinear simulation of turbulence is performed by using parameters; $B=0.1[\mathrm{~T}], T_{e}=3[\mathrm{eV}], a=10[\mathrm{~cm}]$, length of the device $\lambda=4[\mathrm{~m}], v_{\text {in }}=0.035, \mu_{N}=\mu_{V}=$ $10^{-2}, \mu_{U}=10^{-4}$, and the collision frequency $v_{e}=510$. In order to consider a situation when the gradients of the density and the parallel flow coexist, time independent particle and parallel momentum sources are introduced, whose profiles are given as

$$
\begin{aligned}
& S_{n}(r)=\frac{4 S_{N} \mu_{N}}{L_{N}^{2}}\left\{1-\left(\frac{r}{L_{N}}\right)^{2}\right\} \exp \left[-\left(\frac{r}{L_{N}}\right)^{2}\right], \\
& S_{v}(r)=S_{V} \exp \left(-\frac{r^{2}}{L_{V}^{2}}\right),
\end{aligned}
$$

where $S_{N}$ and $S_{V}$ determine the intensity of the sources, and $L_{N}$ and $L_{V}$ give the scale lengths of the mean profiles without fluctuations. In this simulation, $L_{N}=6[\mathrm{~cm}]$ and $L_{V}=3[\mathrm{~cm}]$ are taken. The intensities of the density and parallel momentum sources control the stabilities of the resistive drift wave in the presence of the parallel flow. In
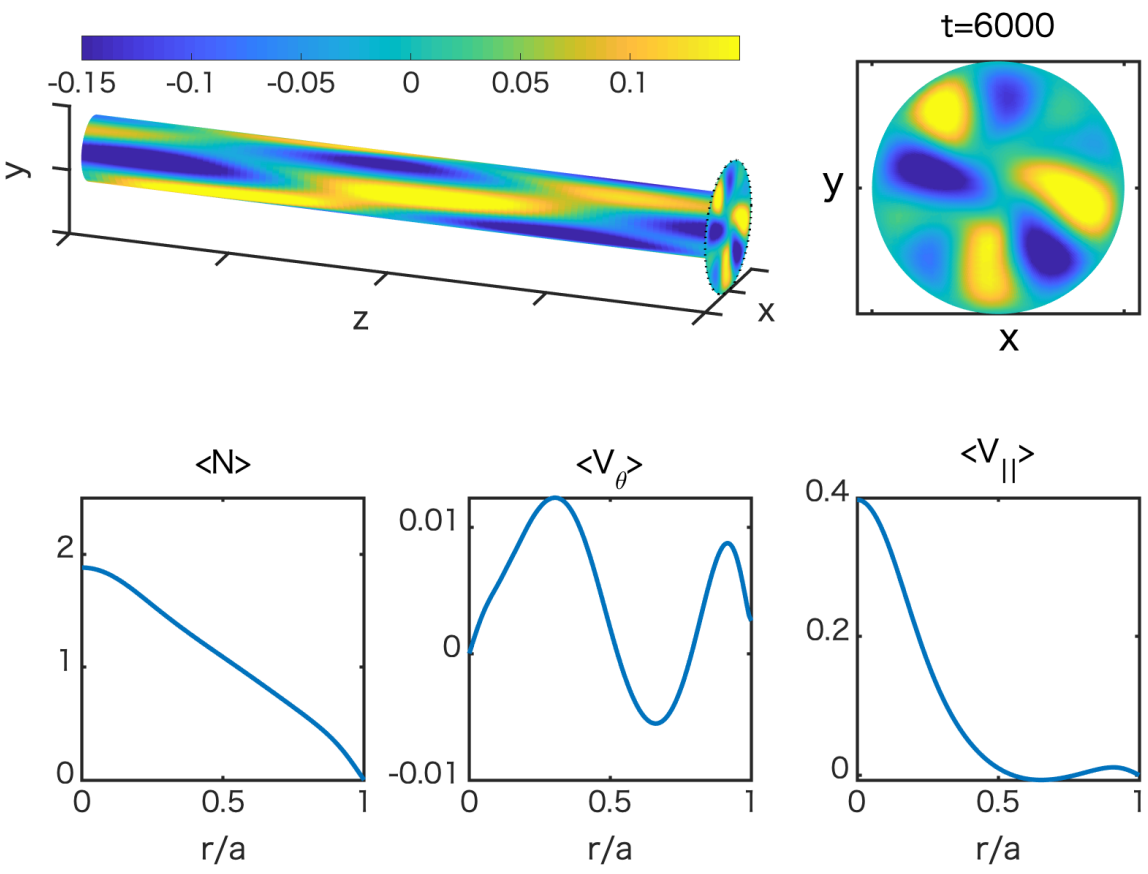

Fig. 1 Outputs of the turbulence simulation in the case of $S_{V}=0.03$. The upper figures show the potential fluctuation pattern: left one is the three dimensional pattern, and the right one is that at $z=0$. The bottom figures illustrate the radial profiles of the background, such as density, the azimuthal flow (which is calculated from the potential profile), and the parallel flow. 
order to study the behavior of the zonal flow in the presence of the parallel flow, the simulations are performed with changing the parallel momentum source intensity $S_{V}$ shot-by-shot with the fixed density source $S_{N}=4$. It is noted that this simulation is the flux-driven simulation so that the background profiles of the density, potential and parallel flow are self-consistently evolved. The time evolution of the three-dimensional fluctuation pattern for the density, the potential and the parallel flow are calculated by using Fourier mode decomposition for the azimuthal and axial directions.

Figure 1 illustrates the snapshot of the turbulence fluctuation and the background profiles. The upper figure shows the potential fluctuation pattern, whose axial wavelength is much longer than the azimuthal wavelength, and which propagates in the electron diamagnetic direction. These are the characteristics of the drift wave [26]. Although the axial phase propagation is symmetry (the magnitudes of the modes with the positive and negative phase velocity are the same) without the parallel flow, the finite parallel flow violates this symmetry and the preferential phase propagation direction appears. This asymmetry in the axial propagation leads to the generation of the parallel momentum flux, which works to suppress the parallel flow shear [25]. At the same time, the fluctuation drives the zonal flow as seen in the bottom center of Fig. 1, where the azimuthal flow is calculated from the potential profile, assuming the $E \times B$ flow, $\left\langle V_{\theta}\right\rangle=\partial_{r}\langle\phi\rangle$. Here, the bracket $\langle\cdots\rangle$ denotes $m=n=0$ component, where $m$ and $n$ are the azimuthal and axial mode numbers, respectively.

\section{Behavior of Zonal Flows in the Presence of Parallel Flows}

In this section, the behavior of the zonal flow is studied when the parallel momentum source changes. First, the change of the background plasma condition with respect to change of the parallel momentum source is discussed, and the zonal flow characteristics are shown. Then, we describe the change of the turbulence properties, and the turbulence effect on the zonal flow is discussed.

The background plasma properties, including the change of the zonal flow, are described. Figure 2 shows the summary of the behaviors of the background. Here, the number of particles stored in the plasmas is calculated from the volume integral of the mean density profile. The kinetic energies in the perpendicular and parallel directions are obtained from $2^{-1} \int \partial_{r}\langle\phi\rangle^{2} r d r$ and $2^{-1} \int\left\langle V_{\|}\right\rangle^{2} r d r$, respectively. The parallel kinetic energy smoothly increases roughly with the square function of $S_{V}$. On the other hand, the perpendicular kinetic energy, which corresponds to the energy of the zonal flow, shows the complex behavior; the energy decreases in the region $S_{V}<0.003$, and increases in $0.003<S_{V}<0.017$. Around $S_{V} \approx 0.017$, the zonal flow energy shows the sudden drop, which corresponds to the transition of the state. The radial profile of $V_{\theta}$ and $V_{\|}$ are shown in Figs. 2 (d) and (e). The profile of the zonal flow changes as follows; inner region of the flow around
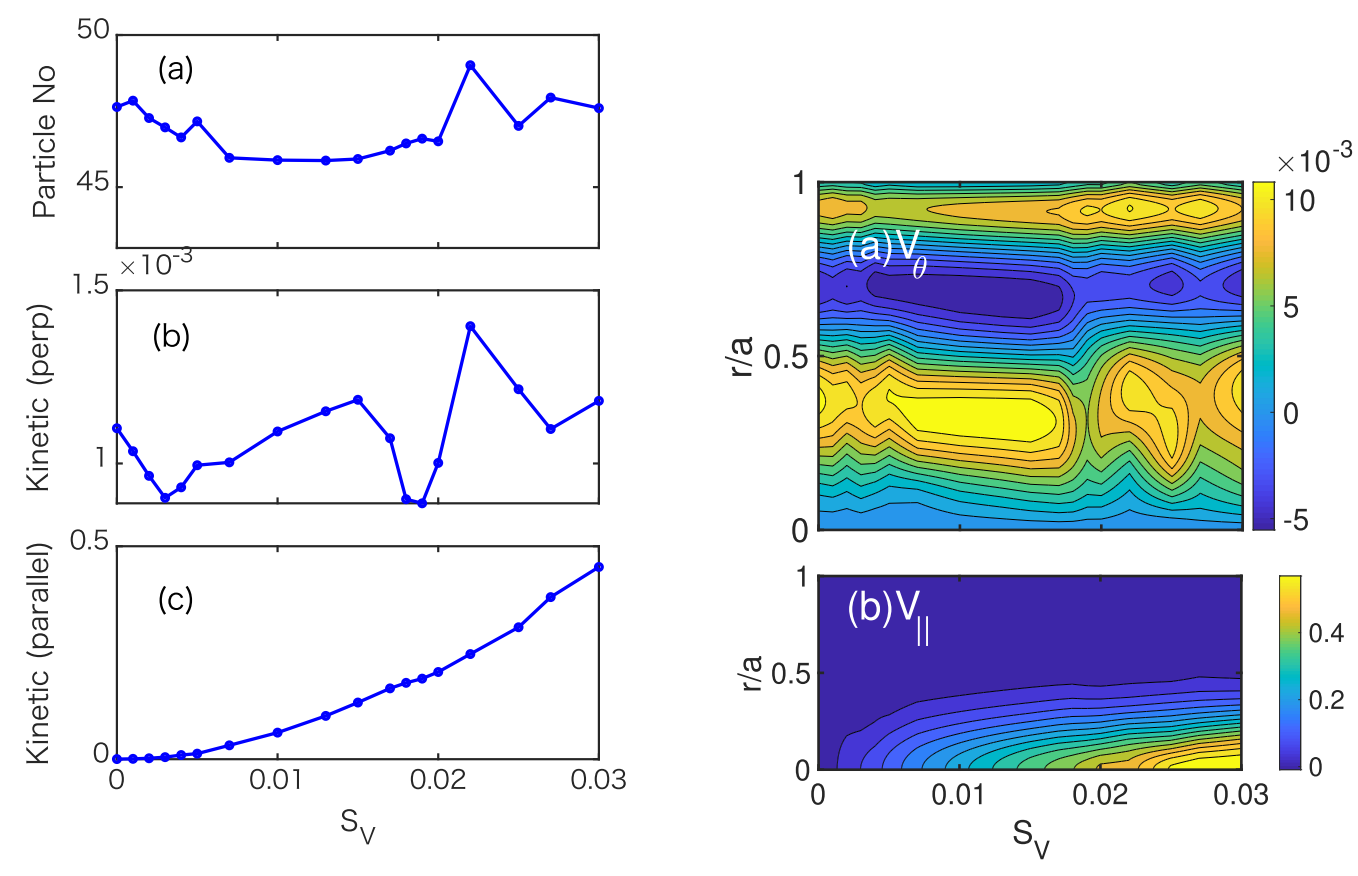

Fig. 2 Changes of background plasmas with respect to the change of the parallel flow; (a) the stored particle number, (b) the perpendicular kinetic energy (zonal flow energy), (c) the parallel kinetic energy (d) the radial profile of the azimuthal flow, and (e) the radial profile of the parallel flow. Here, the each kinetic energy in the perpendicular and parallel directions are calculated from the $m=n=0$ components of the potential and the parallel flow, respectively. 

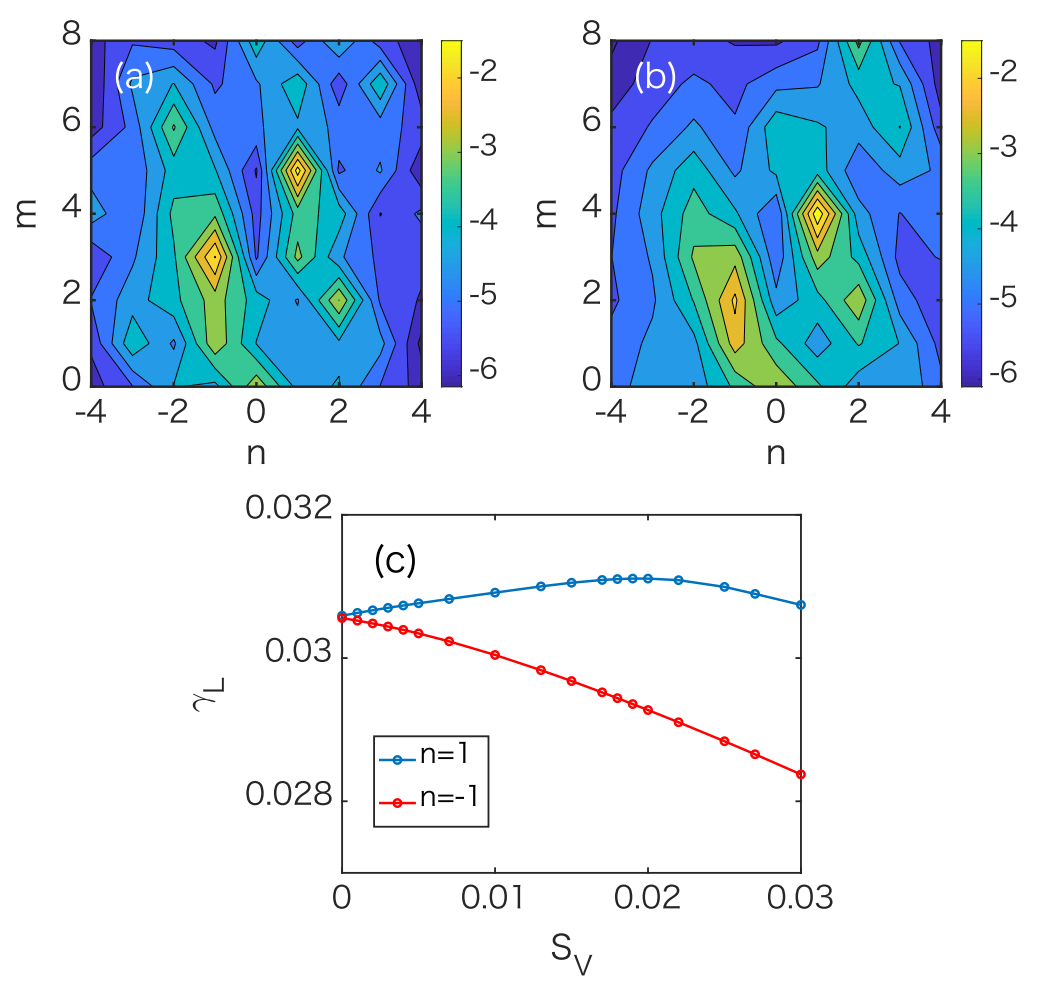

Fig. 3 Wavenumber spectrum of the potential fluctuation in the cases of (a) $S_{V}=0.017$ and (b) $S_{V}=0.03$, and (c) dependence of linear growth rate of the modes on $S_{V}$. Here, $m$ and $n$ are the azimuthal and axial mode numbers, respectively. The intensities of the wavenumber spectrum are plotted in the logarithmic scales. The linear growth rate of the modes with $n= \pm 1$ is evaluated from the time evolution of their energy.

$r / a \sim 0.3$ suddenly weakens at $S_{V}>0.017$, while the flow at outer region $r / a \sim 0.9$ increases at $S_{V}>0.017$. This change in the radial profile is reflected by the change of the volume integral of the perpendicular kinetic energy. The change of the total particles stored in the plasma, Fig. 2 (a), roughly corresponds to the change of the volume integral of the zonal flow energy, Fig. 2 (b). This indicates that the parallel flow itself does not have an impact on the particle transport, but it can contribute to the transport control through the change of the zonal flow.

In order to show the physical mechanism of the zonal flow profile change, the behavior of the fluctuation properties are discussed. Figure 3 illustrates the wavenumber spectrum of the turbulent kinetic energy, calculated from $E_{K}(m, n)=2^{-1} \int\left|\nabla_{\perp} \phi_{m, n}\right|^{2} r d r$, in the cases before and after the transition. In both cases, the parallel wavelength of the dominant modes are comparable to the device, $n= \pm 1$, which is a characteristics of the drift waves. The sign asymmetry in $n$ stems from the parallel flow; the sign of the flow affects the drift wave growth rate and the shear is related to the instability of the D'Angelo mode. The linear growth rate of the drift wave in the presence of the parallel flow, $\gamma_{D W}$, is given as [26]

$$
\gamma_{D W}=\frac{k_{\perp}^{2}\left(-m \partial_{r}\langle N\rangle\right)\left(-m \partial_{r}\langle N\rangle+n(r / \lambda)\left\langle V_{\|}\right\rangle\right)}{D r^{2} n^{2} \lambda^{-2}\left(1+k_{\perp}^{2}\right)^{2}},
$$

where $k_{\perp}$ is the perpendicular wavenumber. When the par- allel flow is present, the growth rate of the mode with $n\left\langle V_{\|}\right\rangle>0$ becomes large, and the growth rate of the mode with $n\left\langle V_{\|}\right\rangle<0$ becomes small. In this study, the positive parallel flow is applied, and, thus, the mode with the positive parallel wavenumber becomes large. It is noted that Eq. (6) is derived by neglecting the shear of the parallel flow, which would change the result. In addition, the drift waves with the opposite sign of $n$ have different effect on the background parallel flow. The role of the modes on the background flow can be understood by looking at the fluctuation induced parallel Reynolds work, $W_{\|}$, which is evaluated as follows [27].

$$
\begin{aligned}
W_{\|} & =-\int\left\langle V_{\|}\right\rangle \frac{1}{r} \partial_{r}\left(r \Pi_{r \|}\right) r d r \\
& \approx-\frac{\lambda}{2 \pi} \sum_{m n} \int \frac{n m \partial_{r}\left\langle V_{\|}\right\rangle}{n^{2} D}\left|\phi_{m n}\right| d r
\end{aligned}
$$

where the azimuthal mode, $m$, is positive in the drift wave case (the fluctuation propagates in the electron diamagnetic direction), and the negative parallel flow shear is formed in the present simulation cases. The sign of the parallel Reynolds work depends on $n m \partial_{r}\left\langle V_{\|}\right\rangle$: the modes with negative/positive $n$ drives the negative/positive work, which suppresses/drives the parallel flow. In this way, the modes with $n=1$ and $n=-1$ have different characteristics, and they nonlinearly compete with each other as reported in [25]. In this study, we consider the situation with the 


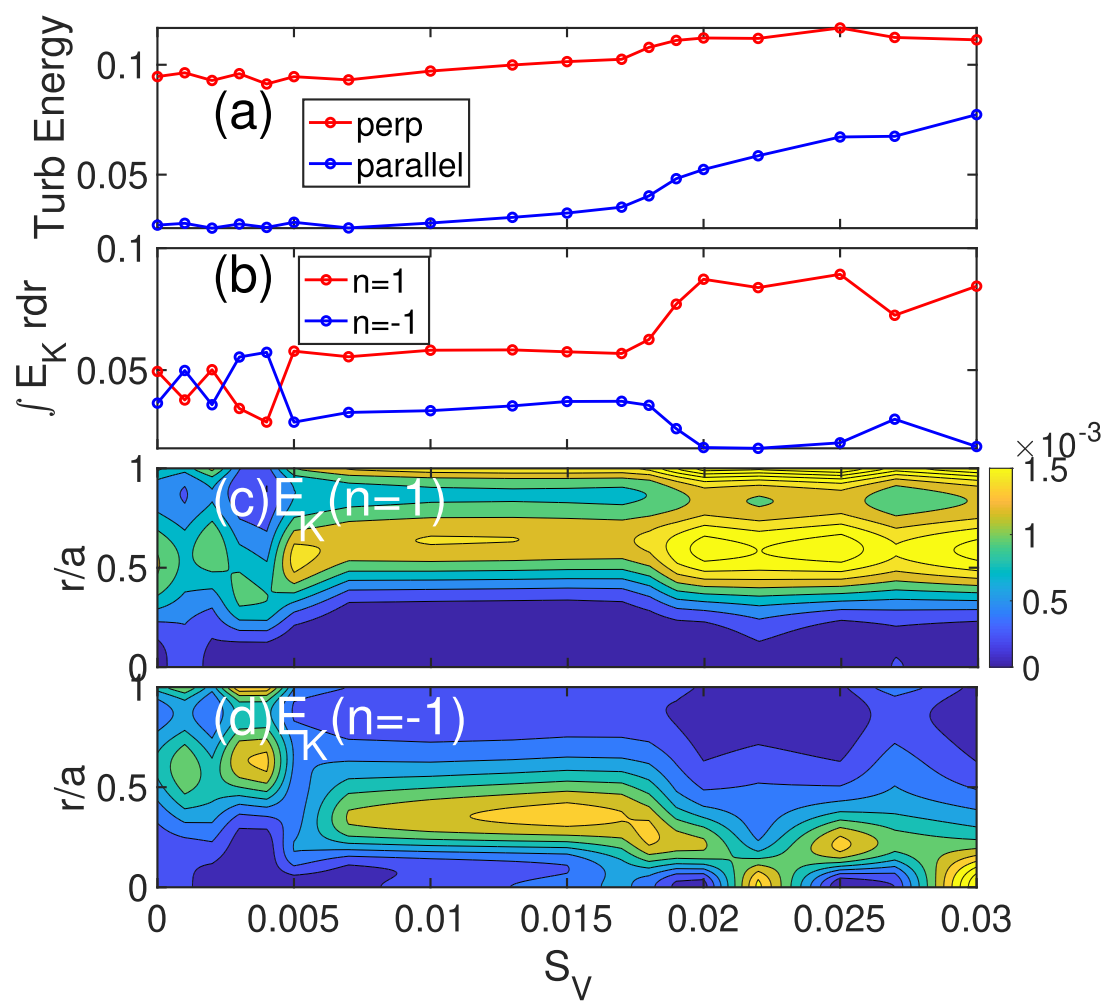

Fig. 4 Changes of fluctuation characteristics with respect to the change of the parallel flow. (a) Volume integral of turbulence kinetic energies, (b) turbulent kinetic energy, (c) radial profile of the turbulent kinetic energy calculated from $n=1$ and (d) radial profile of the turbulent kinetic energy calculated from $n=-1$.

large density gradient, and focus on the drift wave turbulence with the parameter range where the D'Angelo mode is not excited. The linear growth rate of the modes $\gamma_{L}$ with $n= \pm 1$, shown in Fig. 3 (c), are evaluated from the time evolution of the energy as

$$
\gamma_{L}(n)=\frac{1}{T} \int_{0}^{T} \partial_{t}\left(\ln \sum_{m} E_{m n}(t)\right) d t,
$$

where $T$ is chosen to be $T=1800$ (before the nonlinear saturation). Both modes with $n= \pm 1$ are linearly unstable in all cases. Each growth rate is the same in the case of $S_{V}=0$ as described above, and the growth rate with $n=$ 1 mode becomes approximately $10 \%$ larger than that of $n=-1$ mode. In this way, although the linear growth rate smoothly changes depending on the injection of the parallel momentum source, the behavior of the nonlinear saturated amplitude does not show the smooth change but it shows the transition.

Next, we show the radial profile bifurcation of the fluctuation. The changes of the volume integral of the turbulent energy, and its radial profiles with respect to the change of the parallel momentum source are shown in Fig. 4. The perpendicular and parallel turbulence kinetic energies is shown in Fig. 4(a). The parallel kinetic energy is much smaller than that of the perpendicular energy before the transition at $S_{V}<0.017$, and increases at $S_{V}>0.017$ to be comparable to the perpendicular energy.
This indicates that the parallel fluctuation does not contribute much for the background plasma at $S_{V}<0.017$, and becomes important at $S_{V}>0.017$. The volume integral of the kinetic energy with $n= \pm 1$ components, and their radial profiles are shown in Figs. 4(b), (c) and (d), respectively. In the case with the small parallel momentum source at $S_{V}<0.003$, where the mean parallel flow driven by the turbulence and that by the source are comparable, the modes with $n= \pm 1$ competes with each other, and both modes can be dominant. In the region with $0.003<S_{V}<0.017$, although the volume integral of the energies of $n= \pm 1$ modes coexists, the existence spatial region is separated. The peak of the modes with $n=1$ exists around $r / a \approx 0.6$, while the peak of the modes with $n=-1$ is around $r / a \approx 0.4$, which shows the spatial competition. Because the mode with $n=1$ exists in the outer region, the volume integral of the energy is larger than that of the $n=-1$ modes, while the each peak magnitudes are similar. At $S_{V}>0.017$, where the zonal flow energy suddenly changes, the modes with $n= \pm 1$ competes strongly, the modes with $n=-1$ almost disappears, and the existence region of the modes with $n=1$ becomes wider, which leads to the sudden rise in the energy of $n=1$ mode. The disappearance of the spatial competition observed in the region of $0.003<S_{V}<0.017$ abruptly occurs, and the turbulence state transition is obtained. This transition of the state can not be predicted by the local linear sta- 


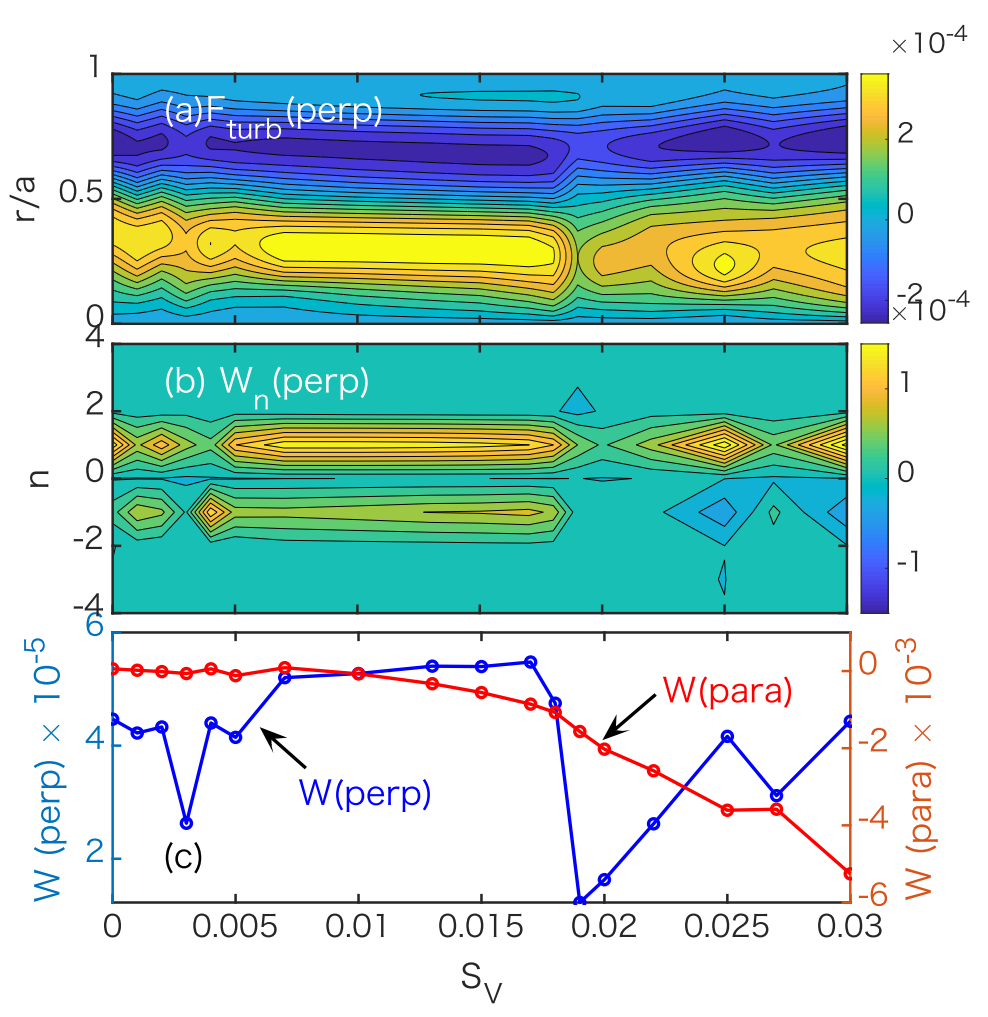

Fig. 5 (a) Change of radial profile of the turbulent forces by the parallel flow momentum source, where the force is calculated from the radial derivative of the perpendicular Reynolds stress. (b) Mode contribution on perpendicular Reynolds work. (c) Changes of the turbulent work to drive the azimuthal and axial flow. Here, the positive/negative sign corresponds to the drive/deceleration.

bility [26] nor the nonlinear analytical theory as in [23]. In the situation where the transition is observed, the linear growth rates of the modes with $n= \pm 1$ are close, so that their contributions to the background profiles are similar. The coupling between the modes with $n= \pm 1$ through the deformation of the background is a possible candidate to explain this transition, which is a future work. In this study, we focus on the mechanism that causes the change of the zonal flow characteristics by the parallel flow shear (as consequences of the appearance/disappearance of the spatial competition between the turbulence modes).

In order to show that the transition of the zonal flow energy at $S_{V} \approx 0.017$ is due to the abrupt change of the turbulence profiles, the Reynolds forces driven by the turbulence are discussed. The Reynolds forces acting on the perpendicular and parallel flows, $F_{\perp}$ and $F_{\|}$are evaluated from $F_{\perp}=-r^{-1} \partial_{r}\left(r \Pi_{r, \theta}\right)$ and $F_{\|}=-r^{-1} \partial_{r}\left(r \Pi_{r, \|}\right)$, where the Reynolds stresses are given by $\Pi_{r, \theta}=-r^{-1}\left\langle\partial_{r} \phi \partial_{\theta} \phi\right\rangle$ and $\Pi_{r, \|}=-r^{-1}\left\langle V_{\|} \partial_{\theta} \phi\right\rangle$. The radial profile of the perpendicular turbulence force, which is the driving force of the zonal flow, is shown in Fig. 5 (a). The spatial pattern is very similar to the flow pattern, so that the zonal flow is found to be driven by the perpendicular Reynolds force. In order to clarify the energy transfer between the flow and the turbulence, the volume integrals of the work acting on the flows, evaluated by $W_{\perp}=\int\left\langle V_{\theta}\right\rangle F_{\perp} r d r$ and $W_{\|}=\int\left\langle V_{\|}\right\rangle F_{\|} r d r$, are shown in Fig. 5 (c). Here, the positive work indicates that the energy is transferred from turbulence to the flow, and vice versa. The perpendicular and parallel works are positive and negative in all range of the state, respectively. So, the zonal flow is driven by the turbulence, and, at the same time, the parallel flow is suppressed by the turbulence. The sudden change of the magnitude of the perpendicular work can be seen at $S_{V} \approx 0.003$ and 0.017 , which correspond to the transitions of the turbulence state. Therefore, the abrupt change of the zonal flow characteristics is found to be due to the turbulence transition, the appearance/disappearance of the spatial competition between the turbulence modes. In order to clarify the role of the $n= \pm 1$ modes on the formation of the zonal flow, the contribution of each mode on the perpendicular Reynolds work is evaluated from $W_{n \perp}=\sum_{m} \int m r^{-1} \operatorname{Im}\left[\phi_{m n}^{*} \partial_{r} \phi_{m n}\right]\left\langle V_{\theta}\right\rangle r d r$, which is shown in Fig. 5 (b). The contributions of the $n= \pm 1$ modes are dominant; the contribution of the $n=1$ mode is positive, which drives the zonal flow, and that of the $n=-1$ mode changes its sign from positive to negative across the transition at $S_{V} \approx 0.017$. The $n=-1$ mode works to drive the zonal flow in $S_{V}<0.017$, and suppresses the zonal flow in the case of $S_{V}>0.017$. Thus, the $n=-1$ mode makes the transition sharper. In this way, the parallel flow affects the properties of the turbulence, and as a result, the characteristics of the zonal flow changes. Thus, this mechanism could be a new control knob of the confinement using the parallel momentum injection through controlling 
the zonal flow.

\section{Summary}

Using the turbulence simulation, it is demonstrated that the zonal flow is controlled by the parallel flow even in the cylindrical plasmas. The reduced fluid model is employed, and the background parallel flow is applied by introducing the parallel momentum source. Changing the magnitude of the momentum source, the behavior of the zonal flow is investigated. The drift wave turbulence is affected by the parallel flow, and it shows the appearance/disappearance of the spatial competition between turbulence modes. This change occurs abruptly. As a consequence of this transition between the turbulence state, the radial profile of the turbulence drastically changes, which leads to the change of the turbulence force to drive the zonal flow. In this way, the parallel flow indirectly affects the zonal flow via changing the properties of the turbulence.

\section{Acknowledgments}

The authors acknowledge stimulating discussion with and strong support by Prof. S.-I. Itoh. The paper is dedicated to her memory. This work was partly supported by a grant-in-aid for scientific research of JSPS KAKENHI Grant Number (JP16K18335, JP17H06089, JP16H02442, JP15H02155, JP15H02335, JP18K03578), the collaboration programs of NIFS (NIFS17KNST122, NIFS18KNST137, NIFS19KNST151), Asada Science Foundation, Progress 100 of Kyushu University (NB80645028), and the RIAM of Kyushu University.
[1] P.H. Diamond et al., Plasma Phys. Control. Fusion 47, R35 (2005).

[2] P.H. Diamond et al., Plasma Phys. 3, 1626 (1991).

[3] S.-I. Itoh et al., Phys. Rev. Lett. 60, 2276 (1988).

[4] H. Biglari et al., Plasma Phys. 2, 1 (1990).

[5] K. Itoh et al., Plasma Phys. Control. Fusion 57, 075008 (2015).

[6] M. Sasaki et al., Phys. Plasmas 25, 012316 (2018).

[7] K. Kamiya et al., Plasma Phys. Control. Fusion 60, 014023 (2017).

[8] T. Kobayashi et al., Sci. Rep. 7, 14971 (2017).

[9] R. Betti et al., Phys. Rev. Lett. 74, 2949 (1995).

[10] O.D. Gurcan et al., Phys. Plasmas 14, 042306 (2007).

[11] P.H. Diamond et al., Nucl. Fusion 53, 104019 (2013).

[12] M. Sasaki et al., Nucl. Fusion 57, 036025 (2017).

[13] K. Itoh et al., Plasma Phys. Control. Fusion 38, 1 (1996).

[14] K. Hallatschek, Plasma Phys. Control. Fusion 49, B137 (2007).

[15] M. Sasaki et al., Nucl. Fusion 59, 066039 (2019).

[16] N. D'Angelo, Phys. Fluids 8, 1748 (1965).

[17] P.J. Catto et al., Phys. Fluids 23, 1719 (1973).

[18] M. Barnes et al., Phys. Rev. Lett. 106, 175004 (2011).

[19] Y. Camenen et al., Phys. Plasmas 23, 022507 (2016).

[20] T.T. Tran et al., Plasma Phys. Control. Fusion 61, 065002 (2019).

[21] J. Ball et al., Plasma Phys. Control. Fusion 61, 064004 (2019).

[22] X. Garbet et al., Phys. Plasmas 6, 3955 (1999).

[23] Y. Kosuga et al., Phys. Plasmas 24, 032304 (2017).

[24] Y. Kosuga et al., Phys. Plasmas 25, 100701 (2018).

[25] M. Sasaki et al., Phys. Plasmas 24, 112103 (2017).

[26] M. Sasaki et al., Plasma Fusion Res. 12, 1401042 (2017).

[27] Y. Kosuga et al., Plasma Fusion Res. 10, 3401024 (2015). 